ARTICLE

DOI: $10.1038 / \mathrm{s} 41467-017-02472-6$

\title{
Ligands with 1,10-phenanthroline scaffold for highly regioselective iron-catalyzed alkene hydrosilylation
}

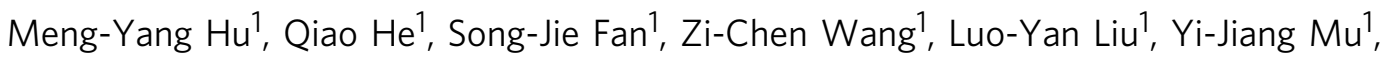 \\ Qian Peng (D) ${ }^{1} \&$ Shou-Fei Zhu (D) ${ }^{1,2}$
}

Transition-metal-catalyzed alkene hydrosilylation is one of the most important homogeneous catalytic reactions, and the development of methods that use base metals, especially iron, as catalysts for this transformation is a growing area of research. However, the limited number of ligand scaffolds applicable for base-metal-catalyzed alkene hydrosilylation has seriously hindered advances in this area. Herein, we report the use of 1,10-phenanthroline ligands in base-metal catalysts for alkene hydrosilylation. In particular, iron catalysts with 2,9-diaryl1,10-phenanthroline ligands exhibit unexpected reactivity and selectivity for hydrosilylation of alkenes, including unique benzylic selectivity with internal alkenes, Markovnikov selectivity with terminal styrenes and 1,3-dienes, and excellent activity toward aliphatic terminal alkenes. According to the mechanistic studies, the unusual benzylic selectivity of this hydrosilylation initiates from $\pi-\pi$ interaction between the phenyl of the alkene and the phenanthroline of the ligand. This ligand scaffold and its unique catalytic model will open possibilities for basemetal-catalyzed hydrosilylation reactions.

\footnotetext{
${ }^{1}$ State Key Laboratory and Institute of Elemento-Organic Chemistry, Tianjin 300071, China. ${ }^{2}$ Collaborative Innovation Center of Chemical Science and Engineering (Tianjin), College of Chemistry, Nankai University, Tianjin 300071, China. Correspondence and requests for materials should be addressed to Q.P. (email: qpeng@nankai.edu.cn) or to S.-F.Z. (email: sfzhu@nankai.edu.cn)
} 
igand design lies at the heart of transition-metal catalysis because ligands play critical roles in the tuning of catalyst activity and selectivity. Although many ligands have been developed, most are based on the same few scaffolds (core structures) ${ }^{1-3}$. The emergence of new types of ligand scaffolds generally revolutionizes the catalytic reactions for which they are used. Therefore, the history of transition-metal catalysis roughly parallels the history of the development of ligands with privileged scaffolds.

Transition-metal-catalyzed alkene hydrosilylation is one of the most important homogeneous catalytic reactions and has been widely used both for basic research and for the industrial production of organosilicons ${ }^{4}$. Precious metals, especially platinum, are the predominant catalysts employed for this transformation ${ }^{5}$, and large amounts of platinum are consumed every year for industrial hydrosilylation of alkenes ${ }^{6}$. The development of methods that instead employ base metals, especially iron, as catalysts is a growing area of research ${ }^{7}$. Base-metal catalysts provide new opportunities for addressing the challenges presented by precious-metal catalysts. The design of suitable ligands is critical to the development of efficient base-metal catalysts. In an elegant example of ligand design, Chirik and co-workers ${ }^{8}$ introduced tridentate bis(imino)pyridine ligands into highly active iron catalysts for selective hydrosilylation reactions of terminal alkenes. This seminal work triggered the design of additional ligands with a pyridine and/or imine scaffold for use in base-metal-catalyzed hydrosilylation reactions ${ }^{9-15}$. However, the number of ligand scaffolds applicable for base-metal-catalyzed alkene hydrosilylation remains limited. This shortage of scaffolds seriously limits the catalytic utility of base metals: the base-metal catalysts developed to date are suitable for the hydrosilylation of terminal alkenes only and cannot be used with internal alkenes. The development of new ligand scaffolds that could overcome this limitation is highly desirable.
Although 1,10-phenanthroline derivatives coordinate strongly to many metals and have been widely used as ligands in transition-metal catalysis ${ }^{16}$, it is surprising that ligands containing this scaffold have never, to our knowledge, been used in alkene hydrosilylation. As part of our ongoing work on ironcatalyzed reactions $^{17-19}$, we herein report the development of bidentate ligands based on a 1,10-phenanthroline scaffold (Darphen, 1) and their use for highly efficient iron-catalyzed alkene hydrosilylation. Catalysts bearing these ligands showed unexpected substrate reactivity and selectivity for iron-catalyzed hydrosilylation of alkenes, including unique benzylic selectivity for internal alkenes, Markovnikov selectivity for terminal styrenes and 1,3-dienes, and excellent activity toward aliphatic terminal alkenes. A set of control experiments and density functional theory (DFT) calculations were also performed to understand the mechanism and to rationalize these unusual selectivities. The mechanistic studies indicate that the benzylic selectivity of this hydrosilylation initiates from $\pi-\pi$ interaction between the phenyl of the alkene and the phenanthroline of the ligand.

\section{Results}

Synthesis of catalysts. We synthesized a series of new iron complexes with 2,9-diaryl-1,10-phenanthroline ligands (Darphen-Fe, 2a-e, Fig. 1a). The corresponding Darphen ligands (1a-e) were easily prepared in high yields from 1,10-phenanthroline by a modified literature procedure ${ }^{20}$. Single-crystal X-ray diffraction analysis (Supplementary Table 1) showed that complex 2c has a distorted tetrahedral structure and contains only one molecule of 1c, which acts as a bidentate ligand. The bulky diaryl groups of 1 may prevent coordination of more than one ligand molecule to the iron center.

Hydrosilylation of alkenes. We began by evaluating the catalytic activity of $\mathbf{2}$ for hydrosilylation of internal alkenes. We chose

a Synthesis of iron complexes of 2,9-diaryl-1,10-phenanthrolines<smiles>Oc1ccc2ccc3ccc(I)nc3c2n1</smiles>

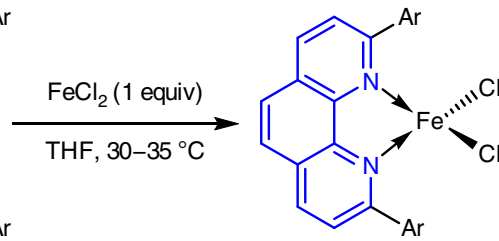

1 (Darphen)

a $\mathrm{Ar}=\mathrm{Ph}$

b $\mathrm{Ar}=3,5-\left({ }^{\mathrm{t}} \mathrm{Bu}\right)_{2} \mathrm{C}_{6} \mathrm{H}_{3}$

c $\mathrm{Ar}=2,4,6-(\mathrm{Me})_{3} \mathrm{C}_{6} \mathrm{H}_{2}$

d $\mathrm{Ar}=2,4,6-(\mathrm{Et})_{3} \mathrm{C}_{6} \mathrm{H}_{2}$

e $\mathrm{Ar}=2,4,6-\left({ }^{\prime} \mathrm{Pr}\right)_{3} \mathrm{C}_{6} \mathrm{H}_{2}$

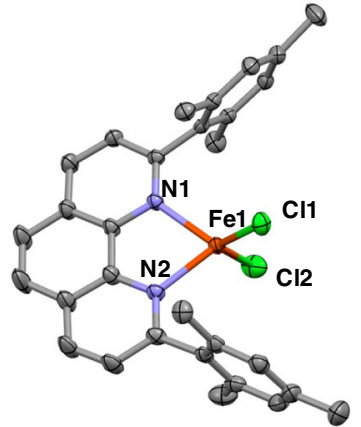

2c (hydrogen atoms and solvent, THF were omitted for clarity): selected bond lengths [Å] and angles [ $\left.{ }^{\circ}\right]$ : Fe1N1 2.1390(16); Fe1-N2 2.1321(16); Fe1-Cl1 2.2440(13); Fe1-Cl2 2.2348(7); N2-Fe1-N1 78.43(6); $\mathrm{Cl} 2-\mathrm{Fe} 1-\mathrm{Cl} 1$ 120.92(3).

b Reference catalysts used in this study<smiles></smiles>

3

$\mathrm{Ar}=2,4,6-\left({ }^{\prime} \mathrm{Pr}\right)_{3} \mathrm{C}_{6} \mathrm{H}_{2}$

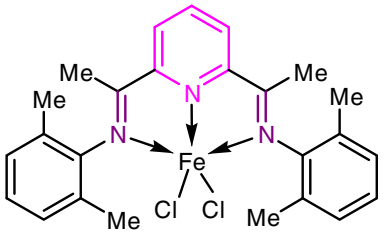

4

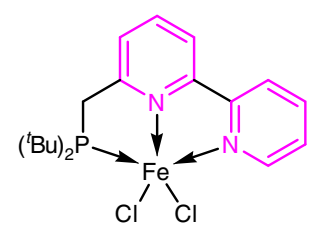

5

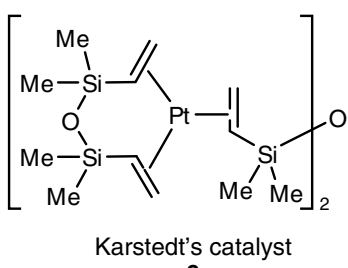

6

Fig. 1 Ligands and catalysts used in this study. a Synthesis of iron complexes of 2,9-diaryl-1,10-phenanthrolines. The diaryl groups in ligand $\mathbf{1}$ may prevent another $\mathbf{1}$ molecule from coordinating with the iron center. $\mathbf{b}$ These catalysts are high-effective catalysts for the hydrosilylation of terminal alkenes 
internal alkenes because although their hydrosilylation has potential utility for the preparation of new organosilicon reagents, however it poses challenges in terms of regioselectivity and substrate reactivity ${ }^{4,7}$. Unlike the two sides of a terminal alkene, the two sides of a dissymmetric internal alkene cannot be readily discriminated. Moreover, the higher steric bulk of internal alkenes hinders hydrosilylation and often favors competitive side reactions, such as isomerization, polymerization, and reduction. For example, although the $\alpha$-hydrosilylation of $\beta$-alkyl styrenes with trichlorosilane, an inorganic silane, has been achieved with palladium catalysts ${ }^{21,22}$, in the presence of base-metal catalysts, internal alkenes undergo rapid alkenyl isomerization to form terminal alkenes before hydrosilylation ${ }^{12,23-26}$.

We chose $\beta$-methyl styrene (7a) as the initial model substrate and phenylsilane as the silylation reagent (Table 1). As a reductant, we used the Grignard reagent EtMgBr, which is thought to reduce iron(II) complexes to active low-valent iron species $^{27}$. Although iron complex 2a, which has a 2,9-phenyl1,10-phenanthroline ligand, was inactive in the hydrosilylation reaction (Table 1 , entry 1 ), complexes $\mathbf{2 b}$-e, which have bulkier aryl groups in the ligands, exclusively afforded the product of benzylic hydrosilylation in moderate to high yields (Table 1, entries 2-5). No other hydrosilylation product was detected in all these cases. The catalyst performance was strongly influenced by the steric bulk of the aryl groups in ligands $\mathbf{1}$, and catalyst $\mathbf{2 e}$, which has a 2,4,6-isopropylphenyl ligand, gave the best results (>95\% conversion and $91 \%$ yield, Table 1 , entry 5 ).
The reaction conditions were then systematically studied. Other organometallic reductants, such as $\mathrm{EtMgCl}, \mathrm{MeMgBr}$, PhMgBr, $\left(p\right.$-Tol)MgBr, ${ }^{n} \mathrm{BuLi}$, and $\mathrm{LiAlH}_{4},{ }^{19}$ gave outcomes similar to those obtained with EtMgBr (Table 1, entries 6-11). Although having similar reactivity, the active catalysts generated from different activators might be different. ${ }^{28-30}$ In contrast, $\mathrm{NaHBEt}_{3}$ and ${ }^{t} \mathrm{BuOK}$ gave lower conversion and yield (Table 1 , entries 12 and 13). When the reaction was performed without solvent, the catalyst loading could be reduced to $1 \mathrm{~mol} \%$, and the reaction could be scaled up to gram scale without diminishing either the yield or the regioselectivity (Table 1, entries 14-16). Although the iron complex was decomposed during the work-up, ligand 1e was recovered in $90 \%$ yield, indicating its high stability under the reaction conditions. The 1,10-phenanthroline scaffold of ligands 1 was critical for the unusual substrate reactivities of the corresponding catalysts. A ligand with a 2,2'-bipyridine scaffold (3, Fig. 1b) was totally inactive under identical reaction conditions (Table 1, entry 17). Iron catalysts with pyridine- and imine-bearing ligands (4 and 5, respectively, Fig. 1b), which efficiently catalyze the hydrosilylation of terminal alkenes ${ }^{7}$, failed to afford the desired hydrosilylation product (Table 1, entries 18 and 19). Note that well-established precious-metal-based hydrosilylation catalysts ${ }^{4}$, such as $\mathrm{H}_{2} \mathrm{PtCl}_{6} \cdot 6 \mathrm{H}_{2} \mathrm{O}$ (Speier's catalyst), 6 (Karstedt's catalyst, Fig. 1b), and a palladium catalyst modified with a phosphine ligand, were inactive for the hydrosilylation of 7a (Table 1, entries 20-22). These control experiments clearly demonstrate the superiority of our iron catalysts with bidentate

Table 1 Transition-metal-catalyzed hydrosilylation of $\beta$-methyl styrene $7 a$ with phenylsilane ${ }^{a}$

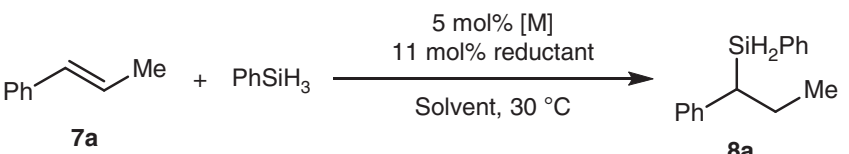

\begin{tabular}{|c|c|c|c|c|c|}
\hline Entry & [M] & Reductant & Solvent & Conv. $(\%)^{b}$ & Yield $(\%)^{c}$ \\
\hline 1 & $2 a$ & $\mathrm{EtMgBr}$ & THF & 0 & ND \\
\hline 2 & $2 \mathbf{b}$ & EtMgBr & THF & 41 & 35 \\
\hline 3 & $2 c$ & EtMgBr & THF & 70 & 65 \\
\hline 4 & $2 d$ & EtMgBr & THF & 88 & 82 \\
\hline 5 & $2 e$ & EtMgBr & THF & $>95$ & 91 \\
\hline 6 & $2 e$ & EtMgCl & THF & $>95$ & 90 \\
\hline 7 & $2 e$ & $\mathrm{MeMgBr}$ & THF & $>95$ & 88 \\
\hline 8 & $2 e$ & $\mathrm{PhMgBr}$ & THF & $>95$ & 90 \\
\hline 9 & $2 e$ & $(p-\mathrm{Tol}) \mathrm{MgBr}$ & THF & $>95$ & 88 \\
\hline 10 & $2 e$ & ${ }^{n} \mathrm{BuLi}$ & THF & $>95$ & 90 \\
\hline 11 & $2 e$ & $\mathrm{LiAlH}_{4}$ & THF & $>95$ & 89 \\
\hline 12 & $2 e$ & $\mathrm{NaHBEt}_{3}$ & THF & 29 & 20 \\
\hline 13 & $2 e$ & ${ }^{t} \mathrm{BuOK}$ & THF & 75 & 68 \\
\hline 14 & $2 e$ & EtMgBr & None & $>95$ & 95 \\
\hline $15^{d}$ & $2 e$ & EtMgBr & None & $>95$ & 95 \\
\hline $16^{e}$ & $2 e$ & EtMgBr & None & $>95$ & 95 \\
\hline 17 & 3 & $\mathrm{EtMgBr}$ & None & 0 & ND \\
\hline 18 & 4 & EtMgBr & None & 0 & ND \\
\hline 19 & 5 & EtMgBr & None & 0 & ND \\
\hline 20 & $\mathrm{H}_{2} \mathrm{PtCl}_{6} \cdot 6 \mathrm{H}_{2} \mathrm{O}$ & None & None & 0 & ND \\
\hline 21 & 6 & None & None & 0 & ND \\
\hline $22^{f}$ & $\mathrm{PdCl}_{2} / \mathrm{MOP}$ & None & None & 0 & ND \\
\hline
\end{tabular}

ND, not determined

Reaction conditions: $7 \mathrm{a} / \mathrm{PhSiH}_{3} /[\mathrm{M}] /$ reductant $=0.25: 0.275: 0.0125: 0.0275(\mathrm{mmol})$, in $1 \mathrm{~mL}$ solvent, at $30{ }^{\circ} \mathrm{C}, 24 \mathrm{~h}$

${ }^{b}$ Conversion of $\mathbf{7 a}$. Determined by ${ }^{1} \mathrm{H}$ NMR using 1,3,5-trimethoxybenzene as internal standard

c Isolated yield

d $\mathbf{7 a} / \mathrm{PhSiH}_{3} / \mathbf{2 e} / \mathrm{EtMgBr}=2: 2 \cdot 2: 0.04: 0.088(\mathrm{mmol})$, at $30^{\circ} \mathrm{C}, 24 \mathrm{~h}$

e Gram-scale experiment: used $5 \mathrm{mmol} \mathbf{7 a}, 5.5 \mathrm{mmol} \mathrm{PhSiH}_{3}, 1 \mathrm{~mol} \% \mathbf{2 e}$, and $2.2 \mathrm{~mol} \% \mathrm{EtMgBr}$, and $1.07 \mathrm{~g}$ of $\mathbf{8 a}$ was obtained

${ }^{f} \mathrm{MOP}=\left(2^{\prime}\right.$-methoxy-[1,1'-binaphthalen $]-2$-yl) diphenylphosphane 


\section{Table 2 Iron-catalyzed hydrosilylation of $\beta$-alkyl styrene with phenylsilane ${ }^{a}$}

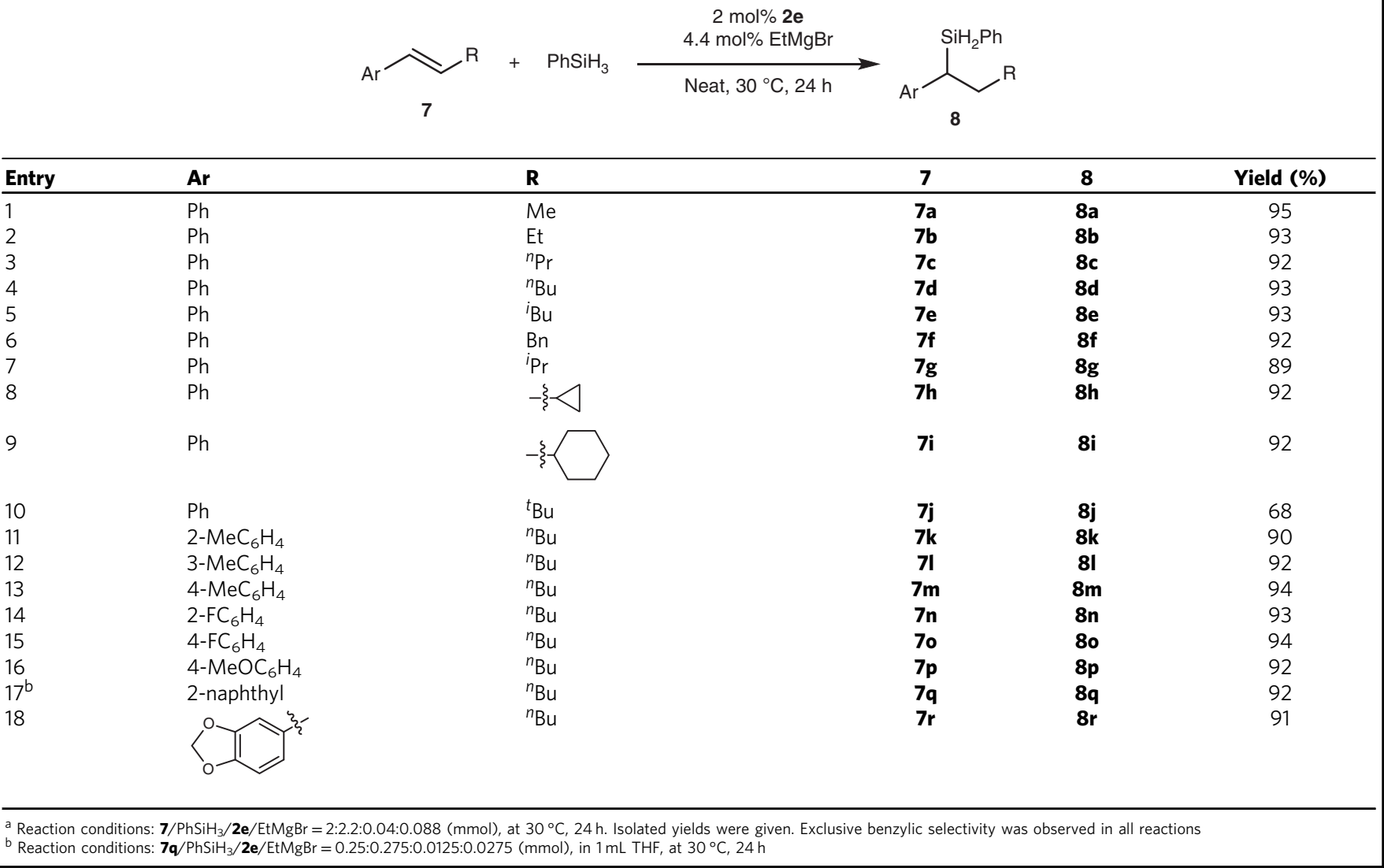

phenanthroline ligands for the hydrosilylation of internal alkenes. Trace metal contamination analysis of iron precatalysts (Supplementary Table 2) and a set of background experiments (Supplementary Table 3) indicate that the iron is the real catalyst in this reaction.

Using catalyst $2 \mathbf{e}$ under the optimized conditions, we then carried out hydrosilylation reactions of various $\beta$-alkyl styrene derivatives 7 (Table 2). First, the effect of the alkyl group $\mathrm{R}$ of the substrate was evaluated (Table 2, entries 1-10). Hydrosilylation reactions of substrates 7 with a wide range of R groups exhibited similar good yields and selectivities (Table 2, entries 1-9). The only exception was $\mathbf{7 j}$, which has a sterically bulky tert-butyl group and gave only a moderate yield of $\mathbf{8 j}$ (Table 2, entry 10). Note that in the reaction of $\mathbf{7 h}$, the highly reactive cyclopropane moiety, which is directly connected to the alkenyl group, remained intact (Table 2, entry 8 ). The steric and electronic properties of the substituents on the benzene ring of the substrates had negligible effects on the reaction outcome; all the tested styrene derivatives (7k-7p) afforded high yields (90-94\%) with exclusive benzylic selectivity (Table 2, entries 11-16). Substrates with fused aromatic ring systems, such as 2vinylnaphthalene $(7 \mathbf{q})$ and 5 -vinylbenzo[ $[d][1,3]$ dioxole $(7 \mathbf{r})$, also gave satisfactory yields and regioselectivities (Table 2, entries 17 and 18 , respectively). We observed the dehydrocoupling of phenylsilane as a side reaction in almost all reactions but with only $<5 \%$ yield. The robustness screen experiments ${ }^{31}$ indicate that the reaction can tolerate ketone, ester, alkyne, borates, and several heteroaromatics, but sensitive to aldehyde, amide, and nitriles (Supplementary Table 4).
Although Markovnikov selectivity has been achieved in the cobalt- and nickel-catalyzed alkene hydrosilylation, ${ }^{13,32-37}$ and in iron- and cobalt-catalyzed alkene hydroboration, ${ }^{38-44}$ the known iron catalysts always exhibit anti-Markovnikov selectivity in the alkene hydrosilylation ${ }^{7}$. To our delight, catalyst $\mathbf{2 b}$ developed in this study showed excellent Markovnikov selectivity ( $\geq 98 \%)$ and high yields (88-95\%) in the hydrosilylation of terminal styrenes 9 (Table 3). The steric and electronic properties of the substituents on the benzene ring of the substrates (9a-9l) had little effect on the reaction outcome. Substrates with fused aromatic ring systems, such as 1-vinylnaphthalene $(\mathbf{9 m}), 2$-vinylnaphthalene (9n), and 5-vinylbenzo[d][1,3] dioxole (9o), also gave satisfactory yields.

We then evaluated the use of iron catalyst $2 \mathbf{e}$ for the hydrosilylation of some other internal alkenes (11) (Table 4). High benzylic selectivity was observed in the hydrosilylation of $1 \mathrm{H}$-indene (11a), a cyclic dissymmetric internal alkene. Surprisingly, the reaction of unconjugated internal alkene $E-\mathbf{1 1 b}$, which has a methylene group between the phenyl and alkenyl moieties, also exhibited good benzylic selectivity (90\%), which suggests that phenyl-directed migration of the double bond occurred before the hydrosilylation. To our knowledge, such reactivity and selectivity toward internal alkenes have not previously been reported. The geometry of the double bond of $\mathbf{1 1 b}$ had little effect on the reaction outcome: a $3: 1 \mathrm{E} / \mathrm{Z}$ mixture of $\mathbf{1 1} \mathbf{b}$ exhibited essentially the same yield and benzylic selectivity as those obtained with pure $E$-11b. When an additional methylene group was introduced between the phenyl and alkenyl moieties (substrate 11c), the hydrosilylation still occurred mainly at the benzyl position, but 


\section{Table 3 Iron-catalyzed hydrosilylation of terminal styrenes ${ }^{a}$}

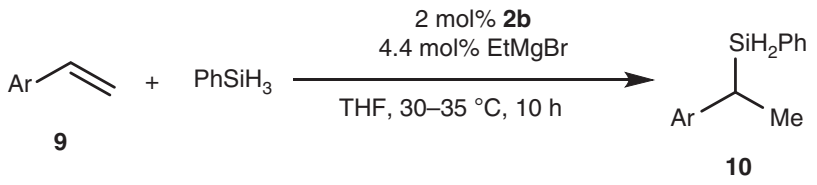

\begin{tabular}{|c|c|c|c|c|}
\hline Entry & Ar & 9 & 10 & Yield (\%) \\
\hline 1 & $\mathrm{Ph}$ & $9 a$ & $10 a$ & 92 \\
\hline 2 & $2-\mathrm{MeC}_{6} \mathrm{H}_{4}$ & $9 b$ & $10 \mathrm{~b}$ & 90 \\
\hline 3 & $2-\mathrm{MeOC}_{6} \mathrm{H}_{4}$ & $9 c$ & $10 c$ & 91 \\
\hline $4^{b}$ & $2-\mathrm{FC}_{6} \mathrm{H}_{4}$ & 9d & 10d & 90 \\
\hline 5 & $3-\mathrm{MeC}_{6} \mathrm{H}_{4}$ & $9 e$ & $10 e$ & 90 \\
\hline 6 & 3- $\mathrm{MeOC}_{6} \mathrm{H}_{4}$ & $9 f$ & $10 f$ & 91 \\
\hline $7^{b}$ & $3-\mathrm{FC}_{6} \mathrm{H}_{4}$ & $9 g$ & $10 \mathrm{~g}$ & 90 \\
\hline $8^{b}$ & $3-\mathrm{ClC}_{6} \mathrm{H}_{4}$ & $9 \mathrm{~h}$ & $10 \mathrm{~h}$ & 90 \\
\hline 9 & $4-\mathrm{MeC}_{6} \mathrm{H}_{4}$ & $9 \mathbf{i}$ & $10 i$ & 91 \\
\hline 10 & $4-\mathrm{MeOC}_{6} \mathrm{H}_{4}$ & 9j & $10 j$ & 93 \\
\hline 11 & $4-\mathrm{FC}_{6} \mathrm{H}_{4}$ & $9 k$ & $10 k$ & 90 \\
\hline $12^{b}$ & $4-\mathrm{ClC}_{6} \mathrm{H}_{4}$ & 91 & 101 & 88 \\
\hline 13 & 1-naphthyl & $9 m$ & $10 \mathrm{~m}$ & 92 \\
\hline 14 & 2-naphthyl & $9 n$ & $10 n$ & 95 \\
\hline $15^{b}$ & Piperonyl & 90 & 100 & 90 \\
\hline
\end{tabular}

a Reaction conditions: $\mathbf{9} / \mathrm{PhSiH}_{3} / \mathbf{2} \mathbf{b} / \mathrm{EtMgBr}=0.5: 0.55: 0.01: 0.022(\mathrm{mmol})$, in $1 \mathrm{~mL} \mathrm{THF}$, at $30-35^{\circ} \mathrm{C}$. The conversion of olefins is $>95 \%$ and the Markovnikov selectivity is $\geq 98 \%$ in all cases ${ }^{b}$ Used $5 \mathrm{~mol} \% \mathbf{2 b}$ and $11 \mathrm{~mol} \% \mathrm{EtMgBr}$

the selectivity was lower (76\%). In contrast, during the reaction of pure alkyl internal alkene 11d, the double bond migrated to the terminus of the molecule, and 12d, which has a terminal silyl group, was obtained as the major product, owing to the lack of the phenyl directing group. Different with its isomer (E)-7a (Table 2, entry 1), the hydrosilylation of (Z)-1-phenyl-1-propene, (Z)-7a was sluggish and afforded a mixture of branched silane (8a) and linear silane (16e) with moderate yield (38\%).

We also evaluated iron catalysts 2 in the hydrosilylation of 1substituted and 1,1-disubstituted buta-1,3-dienes 13 (Fig. 2). Unlike an iron catalyst modified with a tridentate bis(imino) pyridine ligand, which affords a 1:1 mixture of branched and linear products in the hydrosilylation of 1-phenyl-buta-1,3dienes $^{45}$, catalyst 2 c produced 1,2-addition products with exclusive Markovnikov selectivity (14). Again, the substituents on the benzene ring of the 1-aryl-buta-1,3-diene substrates had little effect on the reaction outcome; high yields (91-95\%) of Markovnikov products were observed for all the examined substrates (13a-13f). The hydrosilylation of 1,1-disubstituted buta-1,3-dienes $\mathbf{1 3 g}-\mathbf{1 3} \mathbf{i}$ also proceeded smoothly to give 1,2addition products with exclusive Markovnikov selectivity.

Catalysts 2 also exhibited high activity for the hydrosilylation of 1-alkyl ethylenes 15, but with anti-Markovnikov selectivity (Fig. 3). For example, the reaction of 1-hexene (15a) with phenylsilane in the presence of $0.01 \mathrm{~mol} \% \mathbf{2 e}$ afforded linear hydrosilylated product $\mathbf{1 2 d}$ exclusively in almost quantitative yield. Although the turnover frequency $\left(223 \mathrm{~h}^{-1}\right.$ at $\left.1 \mathrm{~h}\right)$ is moderate comparing to the iron-bis(imino)pyridine catalysts developed by Chirik ${ }^{6}$ and Thomas ${ }^{45}$, the high turnover number (9800) for this reaction indicates that the iron catalyst has a relatively long life time. Functional groups, including chloro (16g), trimethylsilyl (16h), siloxy (16i), amino (16j and 16l), ketal (16k), and quinoline (16l) can be tolerated in this reaction. Moreover, the catalyst also exhibits good tolerances to steric hindrance of the substrates: even highly steric hindered tert-butyl substituted ethylene (15d) can smoothly undergo the hydrosilylation and afford the desired product with satisfactory yield.

This iron-catalyzed hydrosilylation reaction has great potential synthetic utility (Supplementary Fig. 1). For instance, the hydride moiety of silane 8a could readily be transformed to hydroxy, alkoxy, or fluoride in good yield. The silyl group of $\mathbf{8 a}$ could also be directly transformed to other functional groups, such as hydroxyl and chloride. Moreover, the silane products also have potential applications as monomers for preparing polysilanes.

Mechanistic studies. We performed a set of deuteration experiments with alkenes 7a, 9a, and 13a to gain some insight into the reaction mechanism (Fig. 4a). When deuterated silane $\mathrm{PhSiD}_{3}$ was used instead of $\mathrm{PhSiH}_{3}$, no obvious redistribution of the deuterium between the products and the recovered substrates was observed. These results differ from those observed for the hydrosilylation of styrene catalyzed by iron complexes with tridentate bis(imino)pyridine ligands, which shows extensive redistribution of deuterium in the products ${ }^{45}$. Notably, the deuteration experiment with internal alkene $7 \mathbf{a}$ enabled us to uncover the details of the hydrogen transfer processes. The fact that the $\mathrm{D}$ and $\mathrm{Si}$ atoms in product 8a-d were cis to each other (Supplementary Fig. 2) suggests that the addition of silane into the alkene substrate proceeded by means of an inner-sphere coplanar pathway.

A set of kinetic isotopic effect (KIE) experiments were performed through parallel reactions and a secondary KIE effect $\left(k_{\mathrm{H}} / k_{\mathrm{D}}=1.4\right)$ was observed, which indicate that the $\mathrm{C}-\mathrm{H}$ bond formation is not a rate-determining step (Fig. $4 \mathrm{~b}$ and Supplementary Table 5).

Although the efforts on the isolation of single crystal of the active catalysts were unsuccessful, we performed a stoichiometric reduction experiment to determine the oxidation state of the active iron catalyst. When $(p-\mathrm{Tol}) \mathrm{MgBr}$ was used as reductant, 
Table 4 Iron-catalyzed hydrosilylation of other internal alkenes ${ }^{a}$

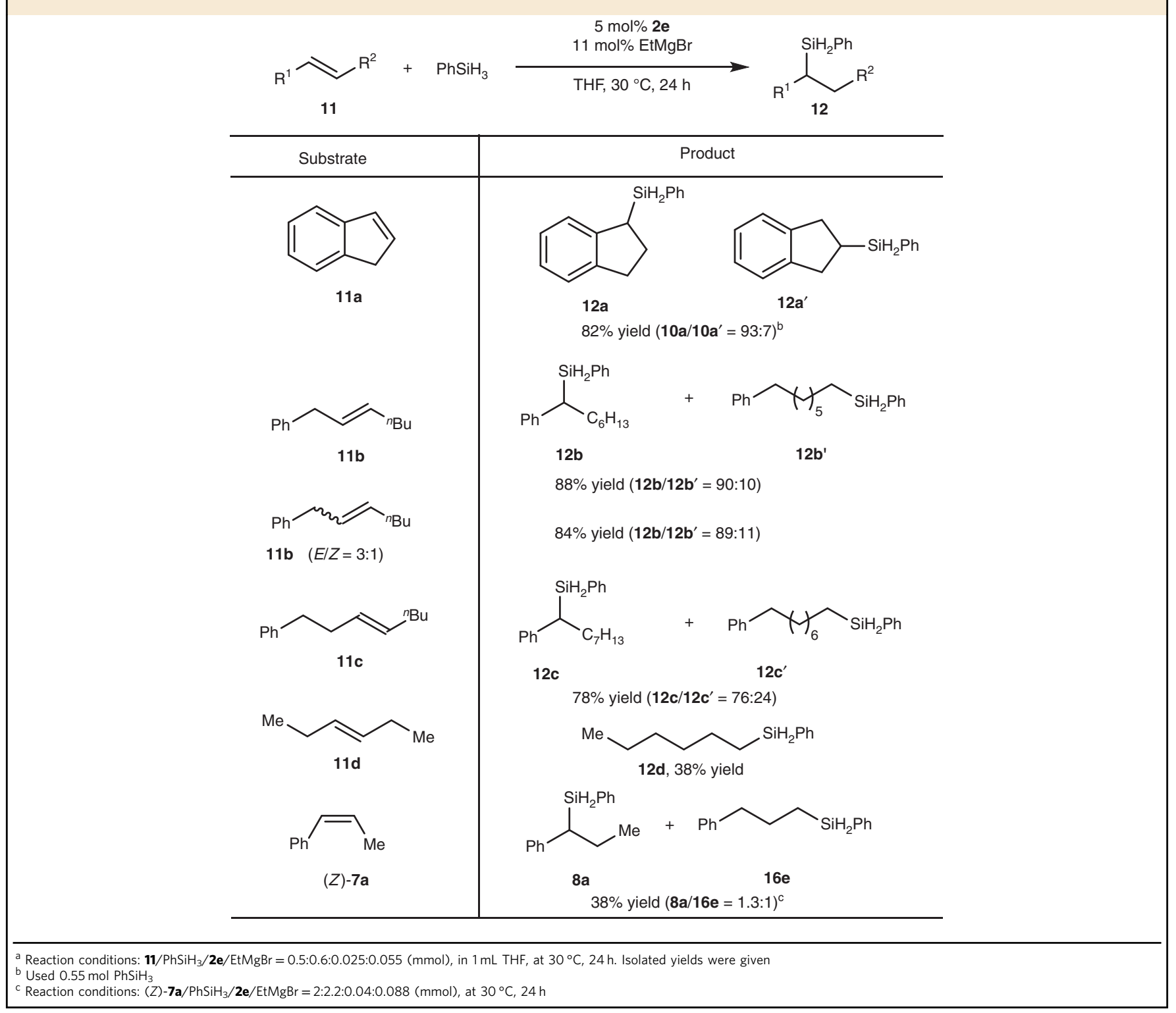

$4,4^{\prime}$-dimethyl-1,1'-biphenyl ( $p$-Tol $)_{2}$ was formed with $48 \%$ yield (based on the Grignard reagent) after quenching the reaction mixture. This experiment implies that a Fe(I) species is most likely generated ${ }^{45}$. The active iron catalyst prepared through this method exhibited the same reactivity for the hydrosilylaion reaction (Table 1, entry 9). Quite recently, Ritter and coworkers $^{46}$ fully characterized a $\mathrm{Fe}(\mathrm{I})$ complex modified with a biimine ligand prepared through the reduction of corresponding $\mathrm{Fe}(\mathrm{II})$ complexes using the Grignard reagent. Considering the similarity of Ritter's procedure and ours, it is reasonable to deduce that the $\mathrm{Fe}(\mathrm{I})$ complex is responsible for the catalytic cycle and play as the real catalyst in this study. We performed an electron paramagnetic resonance (EPR) analysis of the active iron catalyst generated from $\mathrm{Fe}(\mathrm{II})$ and $\mathrm{EtMgBr}$. A strong signal was observed at 3375G (Supplementary Fig. 3), which implies that a high-spin iron species is detected.

According to the above control experiments, we proposed two $\mathrm{Fe}(\mathrm{I})$-catalyzed cycles, which lead to different regioselectivities via hydride insertion (TS-1 or TS-1') and direct Si migration (TS-2 or TS-2') steps (Fig. 5). DFT calculations were introduced to investigate this proposed mechanism in both high-spin $(S=3 / 2)$ and low-spin $(S=1 / 2)$ states at the (dispersion-corrected) unrestricted $\omega \mathrm{B} 97 \mathrm{XD} / 6-31 \mathrm{G}^{*} / \mathrm{TZVP}$ level of theory with

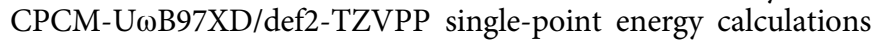
(see Supplementary Tables 6-27 and Supplementary Fig. 4 for details). The $\mathrm{Fe}(\mathrm{I})$ complex in low-spin state is ruled out because the calculated energy is more than $22 \mathrm{kcal} / \mathrm{mol}$ higher than that in the high-spin $\mathrm{Fe}(\mathrm{I})$. This result can be supported by the EPR experiment. The calculations also indicate all the $d$ orbitals are occupied and only $4 s / 4 p$ orbitals are available for coordination in the $\mathrm{Fe}(\mathrm{I})$ complex. The $\mathrm{Fe}(\mathrm{I})-\mathrm{H}$ complex with $\beta$-methyl styrene (7a) coordination formed two distorted tetrahedral structures Int-1 and Int-1' depending on the phenyl orientation of $7 \mathbf{a}$ toward the phenanthroline ligand or not, which initiate two plausible catalytic cycles $\mathrm{A}$ and $\mathrm{B}$. The $\pi-\pi$ interaction between the phenyl of $7 \mathbf{a}$ and phenanthroline ring of the catalyst $(3.68 \AA$ distance) in Int-1 leads to favorable energy by $0.8 \mathrm{kcal} / \mathrm{mol}$ comparing to Int-1'. Hydride insertion into the alkyl end of the double bond is facile, via TS-1 and TS-1' with an activation barrier 19.2 and $21.1 \mathrm{kcal} / \mathrm{mol}$, respectively, forming the stable 


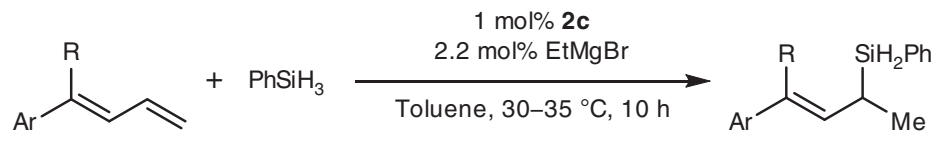

13

14

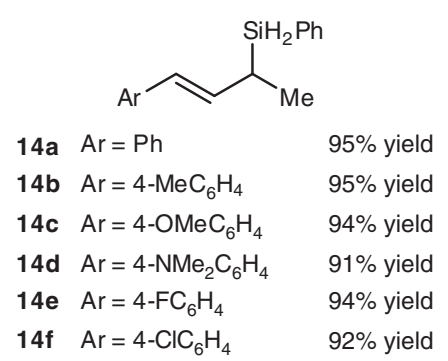<smiles>CC(C=C([Mg])c1ccccc1)C(c1ccccc1)[N+](=O)[O-]</smiles>

$14 \mathrm{~g}, 93 \%$ yield<smiles>CC(C=C(c1ccccc1)c1ccccc1)c1ccccc1</smiles>

$14 \mathbf{i}, 90 \%$ yield

Fig. 2 Iron-catalyzed hydrosilylation of 1-substituted and 1,1-disubstituted buta-1,3-dienes. High yields (91-95\%) with unique Markovnikov selectivities were observed for all the examined conjugated dienes

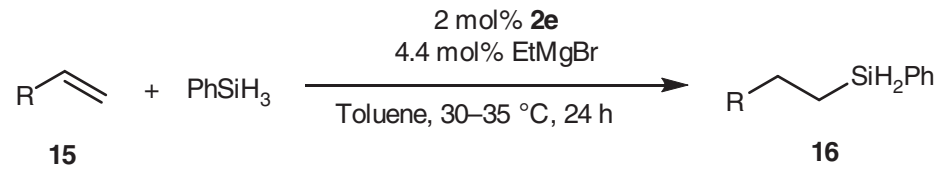

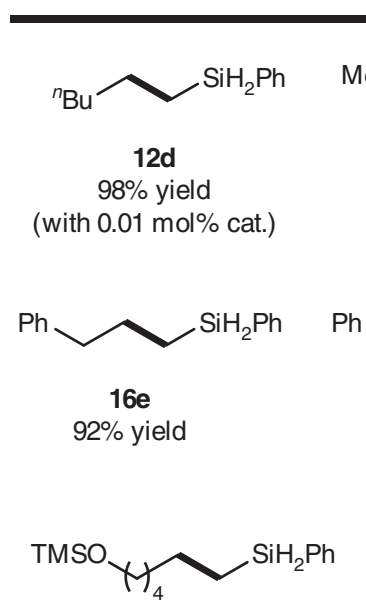

$16 \mathbf{i}$

$93 \%$ yield

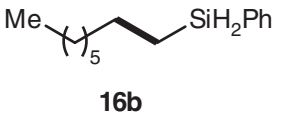

$94 \%$ yield

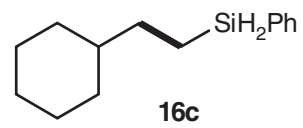

$92 \%$ yield<smiles>CC/C(=C/C([Hg])c1ccccc1)c1ccccc1</smiles>

$14 \mathrm{~h}, 96 \%$ yield

\section{$16 d$}

$90 \%$ yield

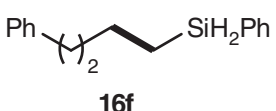

$16 f$<smiles>Cl[Al]CC[Sn]c1ccccc1</smiles>

$16 \mathrm{~g}$

$93 \%$ yield
TMS $\mathrm{SiH}_{2} \mathrm{Ph}$

$16 \mathrm{~h}$

$82 \%$ yield<smiles>COc1ccc2nccc([C@@H](O[Na])C3CC4CCN3C4)c2c1</smiles>

Fig. 3 Iron-catalyzed hydrosilylation of 1-alkyl ethylenes. Exclusive linear products were obtained with remarkable high turnover numbers (up to 9800 ). The reaction exhibits good tolerance to functional groups and to steric hindrance

intermediates Int-2 and Int-2'. The calculated TS-1 and TS-1' were located to coplanar four-member-ring structures, suggesting an inner-sphere coplanar migration in agreement with the cissubstituent D and Si atoms of product 8a-d from our deuteration experiment (Fig. 4a). This migratory insertion is predicted to be irreversible because the barrier in reverse direction is higher by $12.6 \mathrm{kcal} / \mathrm{mol}$ (total $31.8 \mathrm{kcal} / \mathrm{mol}$ for the benzylic selectivity pathway, catalytic cycle A), and therefore it determines the regioselectivity, which rationalize the experimental observation for no obvious redistribution of the deuterium (Fig. 4a). However, our kinetic isotope experiment $\left(k_{\mathrm{H}} / k_{\mathrm{D}}=1.4\right)$ indicates that the hydride insertion is not rate-determining step. Our calculations predict that direct $\mathrm{Si}$ migration is responsible for ratedetermining step with $27.6 \mathrm{kcal} / \mathrm{mol}$ barrier, and it proceeds via four-membered transition state, forming products and regenerating $\mathrm{Fe}(\mathrm{I})$ catalyst by $7 \mathrm{a}$ coordination. The $\mathrm{Fe}-\mathrm{Ph}$ coordination 
$\mathbf{a}_{\text {Deuteration experiments }}$

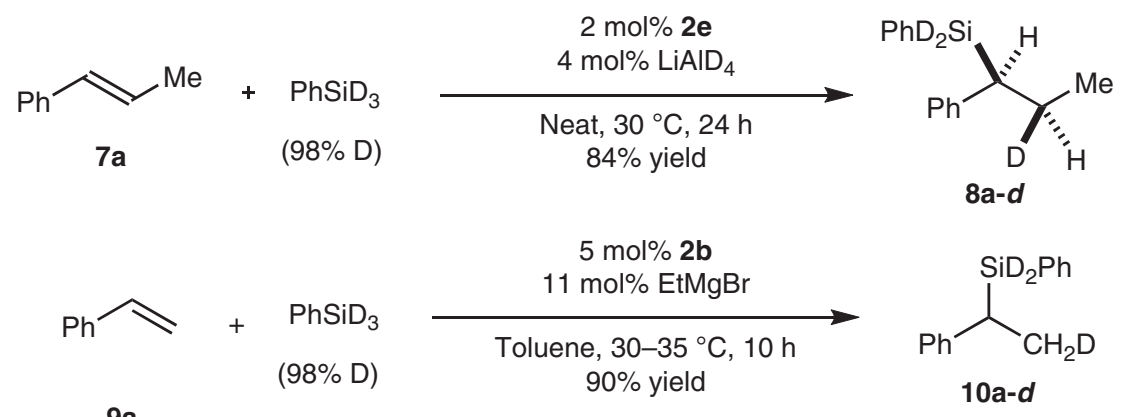

$9 a$

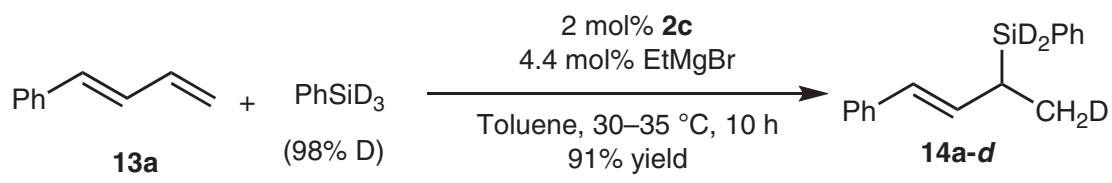

b Kinetic isotopic effect experiments

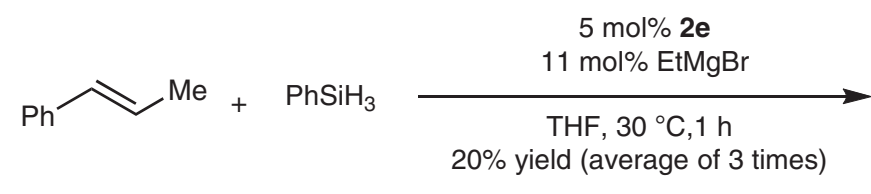

$7 a$<smiles>C[C@H]([SiH3])C(c1ccccc1)c1ccccc1</smiles>

$8 a$

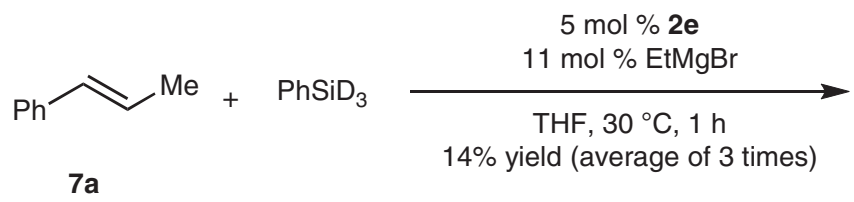

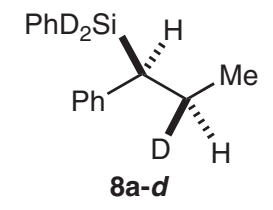

Average $k_{\mathrm{H}} / k_{\mathrm{D}}=1.4$

C Reduction experiment

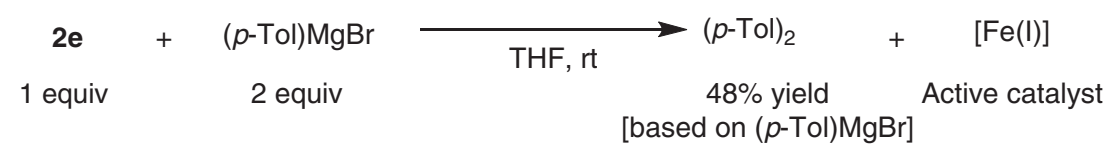

Fig. 4 Control experiments. a Deuteration experiments. The D incorporation in the products are $>95 \%$ in all cases according to ${ }^{1} \mathrm{H}$ NMR. $\mathbf{b}$ Kinetic isotopic effect (KIE) experiments. A secondary KIE effect (average $k_{H} / k_{D}=1.4$ ) was observed. c Reduction experiment. The stoichiometric reduction experiment implies that a $\mathrm{Fe}(\mathrm{I})$ species is most likely generated as the active catalyst

(Fe-C $2.26 \AA$ ) is enabled to stabilize TS-2 and promotes the reaction affording benzylic selective product (Fig. 5). Furthermore, we extended our calculated mechanism to other substrates and the predicted $b / l$ selectivities show good consistence with our experimental results (Table 5).

\section{Discussion}

In summary, we developed iron complex catalysts that have 2,9diaryl-1,10-phenanthroline ligands and exhibit benzylic selectivity in the hydrosilylation of internal alkenes. Reactions of styrenes and 1,3-dienes showed unique Markovnikov selectivity, and these newly developed catalysts also showed excellent activity for the reactions of aliphatic terminal alkenes. Control experiments clearly showed that the 1,10-phenanthroline scaffold of ligands 1 was the basis of the unusual reactivity and selectivity of the catalysts. The introduction of 1,10-phenanthroline as a ligand scaffold for base-metal-catalyzed alkene hydrosilylation has great potential for bringing about significant advances of this area. The unusual catalytic activity associated with the phenanthroline ligands reported herein will inspire the design of additional related catalysts.

\section{Methods}

General methods. See Supplementary Methods for further details.

Typical procedure for hydrosilylation of 7a. In an argon-filled glovebox, a vial (4 $\mathrm{mL})$ was charged with $7 \mathbf{a}(2 \mathrm{mmol}), \mathrm{PhSiH}_{3}(2.2 \mathrm{mmol})$, and complex $2 \mathrm{e}(0.04$ $\mathrm{mmol})$. The reaction mixture was stirred at room temperature $\left(25-35^{\circ} \mathrm{C}\right)$ for 1 min, then EtMgBr ( $1 \mathrm{M}$ in THF, $88 \mu \mathrm{L}, 0.088 \mathrm{mmol}, 4.4 \mathrm{~mol} \%)$ was added. After stirring for $24 \mathrm{~h}$ at $30^{\circ} \mathrm{C}$, the vial was removed from the glovebox and the reaction mixture was concentrated by rotating evaporation. The residue was purified by flash chromatography (hexane) to afford the desired product $8 \mathbf{a}(430.2 \mathrm{mg}, 95 \%)$. ${ }^{1} \mathrm{H}$ NMR (400 MHz, $\mathrm{CDCl}_{3}$ ) $\delta 7.42-7.34$ (m, 3H, Ar-H), 7.33-7.20 (m, 4H, Ar-H), $7.12(\mathrm{t}, J=7.3 \mathrm{~Hz}, 1 \mathrm{H}, \mathrm{Ar}-\mathrm{H}), 7.05(\mathrm{~d}, J=7.3 \mathrm{~Hz}, 2 \mathrm{H}, \mathrm{Ar}-\mathrm{H}), 4.37-4.24$ (m, 2H, Si$\mathrm{H}), 2.40-2.31(\mathrm{~m}, 1 \mathrm{H}, \mathrm{CH}), 1.97-1.79\left(\mathrm{~m}, 2 \mathrm{H}, \mathrm{CH}_{2}\right), 0.90\left(\mathrm{t}, J=7.2 \mathrm{~Hz}, 3 \mathrm{H}, \mathrm{CH}_{3}\right)$; ${ }^{13} \mathrm{C}$ NMR (101 MHz, $\mathrm{CDCl}_{3}$ ) $\delta 142.8$ (1C, Ar-C), 135.7 (2C, Ar-C), 131.4 (1C, ArC), 129.7 (1C, Ar-C), 128.3 (2C, Ar-C), 127.9 (2C, Ar-C), 127.8 (2C, Ar-C), 125.0 (1C, Ar-C), 34.3(1C, CH), $24.5\left(1 \mathrm{C}, \mathrm{CH}_{2}\right), 13.9\left(1 \mathrm{C}, \mathrm{CH}_{3}\right) ;{ }^{29} \mathrm{Si}$ NMR $(79 \mathrm{MHz}$, 

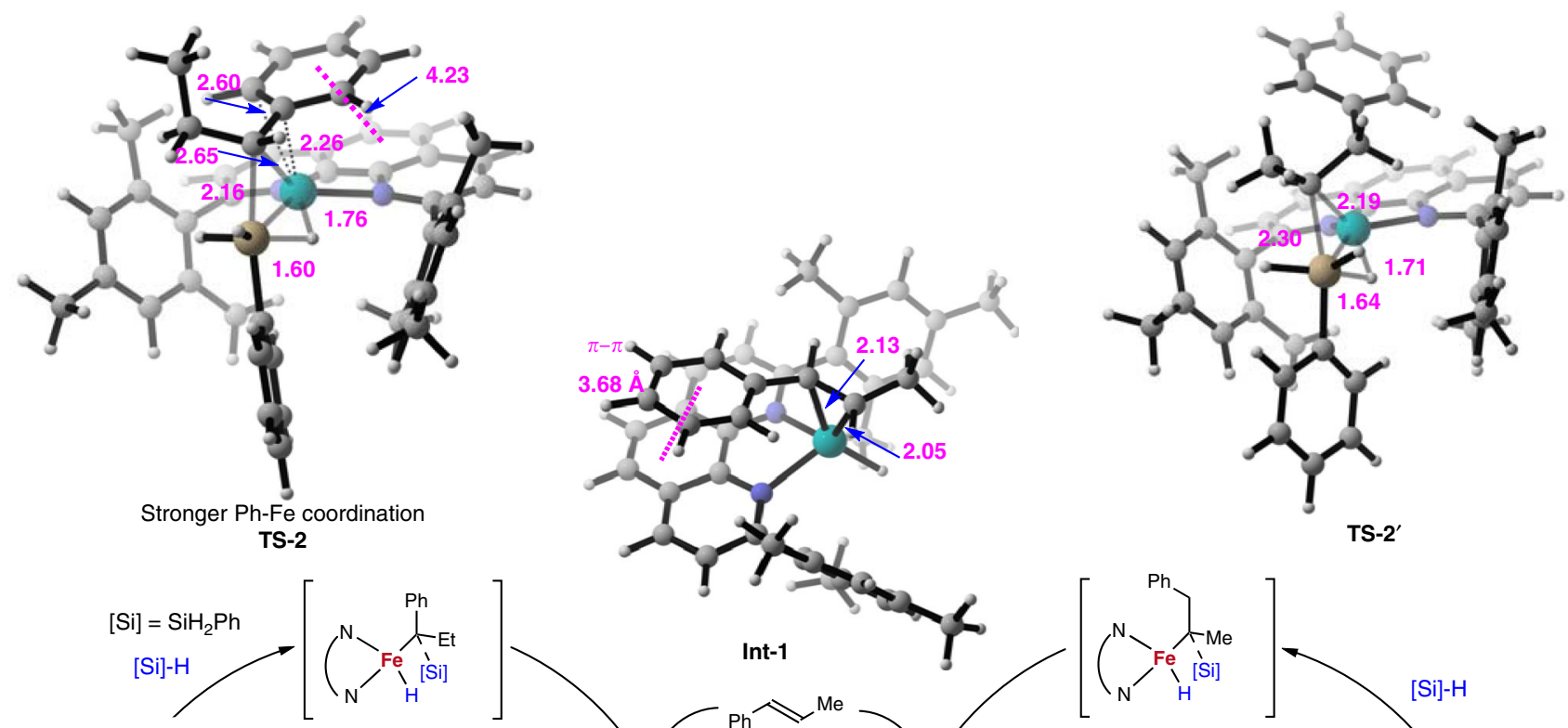

TS-2

Rate-determining step

/

$(+15.0 \mathrm{kcal} / \mathrm{mol})$

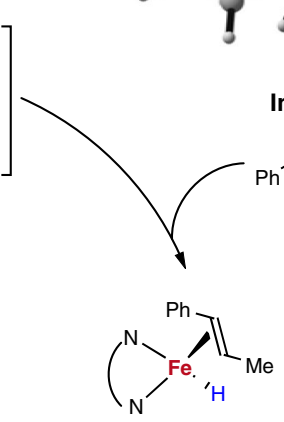

catalytic cycle $A$<smiles>CCC(c1ccccc1)P1NCCN1</smiles>
for $\alpha$-selectivity

Int-2 $(-12.6 \mathrm{kcal} / \mathrm{mol})$

$$
\text { for } \alpha \text { selectivity }
$$
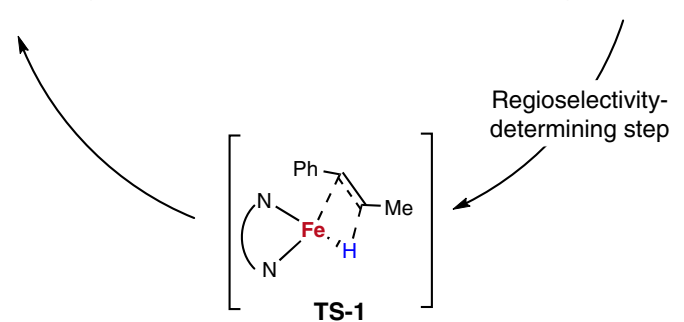

$(+19.2 \mathrm{kcal} / \mathrm{mol})$

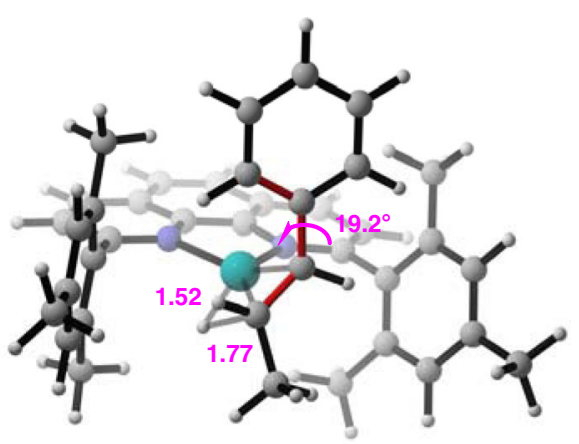

Stronger Ph- $\pi$ conjugation

TS-1

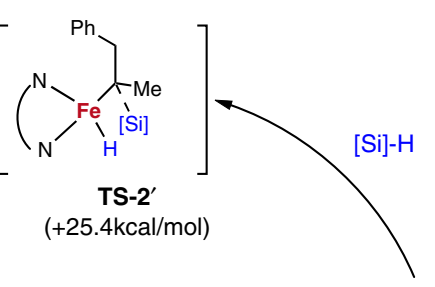
catalytic cycle B for $\beta$-selectivity

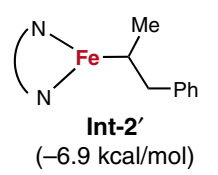

$(+21.1 \mathrm{kcal} / \mathrm{mol})$

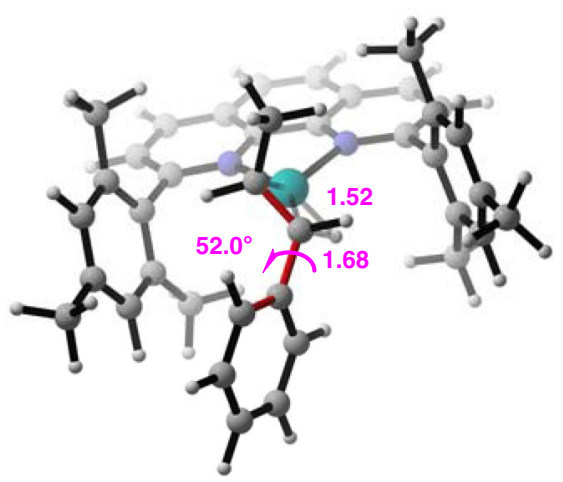

TS-1'

Fig. 5 Computation studies on the mechanism. The catalytic cycles are based on high-spin Fe(I) catalysts. The migratory insertion of Fe-H to alkene is irreversible and determines the regioselectivity. The Si migration is the rate-determining step and passes through four-membered transition state. The benzylic selectivity ( $\alpha$-selectivity) initiates from $\pi-\pi$ interaction between the phenyl of the alkene and the phenanthroline of the ligand

$\left.\mathrm{CDCl}_{3}\right) \delta-20.5,-20.7$. HRMS (EI) calcd for $\left[\mathrm{M}, \mathrm{C}_{15} \mathrm{H}_{18} \mathrm{Si}\right]^{+}:$226.1178; found 226.1180 .

Data availability. Additional data supporting the findings described in this manuscript are available in the Supplementary Information. For full characterization data of new compounds and experimental details, see Supplementary Methods. For the ${ }^{1} \mathrm{H}$ and ${ }^{13} \mathrm{C}$ NMR spectra of new compounds, see Supplementary
Figs. 5-146. Metrical parameters for $2 \mathrm{c}$ are available free of charge from the Cambridge Crystallographic Data Centre under reference numbers CCDC1455690. All other data are available from the authors upon reasonable request.

Received: 4 May 2017 Accepted: 1 December 2017

Published online: 15 January 2018 


\section{Table 5 Regioselectivity of iron-catalyzed alkene hydrosilylation}

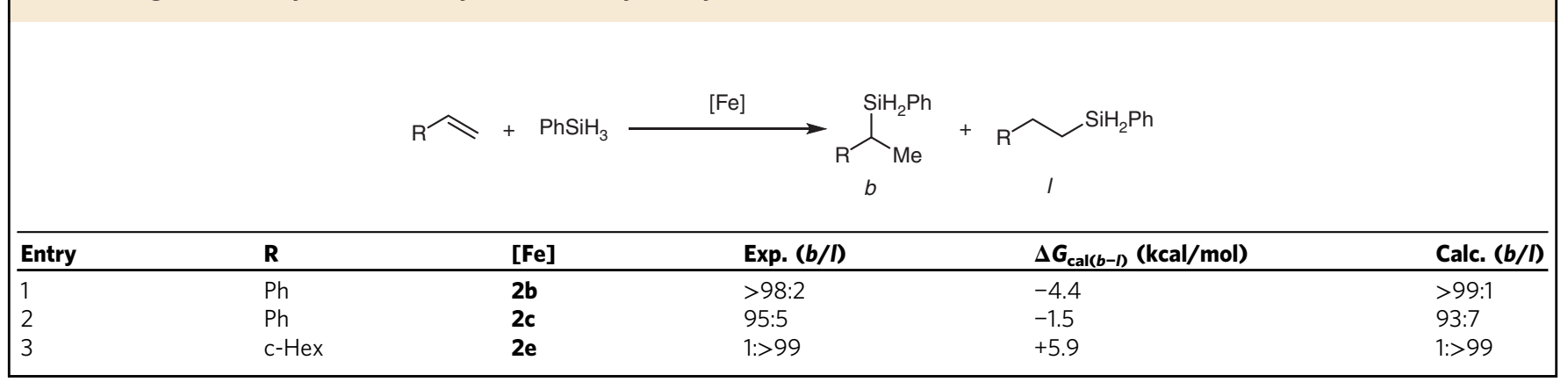

\section{References}

1. Stradiotto, M. \& Lundgren, R. (eds) Ligand Design in Metal Chemistry (Wiley$\mathrm{VCH}, 2016)$.

2. Yoon, T. P. \& Jacobsen, E. N. Privileged chiral catalysts. Science 299, 1691-1693 (2003).

3. Zhou, Q.-L. (ed.) Privileged Chiral Ligands and Catalysts (Wiley-VCH, 2011).

4. Marciniec, B. (ed.) Hydrosilylation, A Comprehensive Review on Recent Advances (Spring, 2009).

5. Markó, I. E. et al. Selective and efficient platinum(0)-carbene complexes as hydrosilylation catalysts. Science 298, 204-206 (2002).

6. Tondreau, A. M. et al. Iron catalysts for selective anti-Markovnikov alkene hydrosilylation using tertiary silanes. Science 335, 567-570 (2012).

7. Du, X. \& Huang, Z. Advances in base-metal-catalyzed alkene hydrosilylation. ACS Catal. 7, 1227-1243 (2017).

8. Bart, S. C., Lobkovsky, E. \& Chirik, P. J. Preparation and molecular and electronic structures of iron $(0)$ dinitrogen and silane complexes and their application to catalytic hydrogenation and hydrosilation. J. Am. Chem. Soc. 126, 13794-13807 (2004)

9. Wu, J. Y., Stanzl, B. N. \& Ritter, T. A strategy for the synthesis of well-defined iron catalysts and application to regioselective diene hydrosilylation. J. Am. Chem. Soc. 132, 13214-13216 (2010).

10. Peng, D. et al. Phosphinite-iminopyridine iron catalysts for chemoselective alkene hydrosilylation. J. Am. Chem. Soc. 135, 19154-19166 (2013).

11. Chen, J., Cheng, B., Cao, M. \& Lu, Z. Iron-catalyzed asymmetric hydrosilylation of 1,1-disubstituted alkenes. Angew. Chem. Int. Ed. 54, 4661-4664 (2015).

12. Chen, C. et al. Rapid, regioconvergent, solvent-free alkene hydrosilylation with a cobalt catalyst. J. Am. Chem. Soc. 137, 13244-13247 (2015).

13. Du, X., Zhang, Y., Peng, D. \& Huang, Z. Base metal-catalyzed regiodivergent alkene hydrosilylations. Angew. Chem. Int. Ed. 55, 6671-6675 (2016).

14. Pappas, I., Treacy, S. \& Chirik, P. J. Alkene hydrosilylation using tertiary silanes with $\alpha$-diimine nickel catalysts. Redox-active ligands promote a distinct mechanistic pathway from platinum catalysts. ACS Catal. 6, 4105-4109 (2016).

15. Toya, Y., Hayasaka, K. \& Nakazawa, H. Hydrosilylation of olefins catalyzed by iron complexes bearing ketimine-type iminobipyridine ligands. Organometallics 36, 1727-1735 (2017).

16. Schoffers, E. Reinventing phenanthroline ligands-chiral derivatives for asymmetric catalysis. Eur. J. Org. Chem. 2003, 1145-1152 (2003).

17. Zhu, S.-F., Cai, Y., Mao, H.-X., Xie, J.-H. \& Zhou, Q.-L. Enantioselective ironcatalysed O-H bond Insertions. Nat. Chem. 2, 546-551 (2010).

18. Shen, J.-J. et al. Enantioselective iron-catalyzed intramolecular cyclopropanation reactions. Angew. Chem. Int. Ed. 53, 13188-13191 (2014).

19. Guo, N., Hu, M.-Y., Feng, Y. \& Zhu, S.-F. Highly efficient and practical hydrogenation of olefins catalyzed by in situ generated iron complex catalysts. Org. Chem. Front. 2, 692-696 (2015).

20. Kuritani, M., Tashiro, S. \& Shionoya, M. Heterodinuclear metal arrangement in a flat macrocycle with two chemically-equivalent metal chelating sites. Inorg. Chem. 51, 1508-1515 (2012).

21. Kitayama, K., Uozumi, Y. \& Hayashi, T. Palladium-catalysed asymmetric hydrosilylation of styrenes with a new chiral monodentate phosphine ligand. J. Chem. Soc. Chem. Commun. 1533-1534 (1995).

22. Jensen, J. F., Svendsen, B. Y., la Cour, T. V., Pedersen, H. L. \& Johannsen, M. Highly enantioselective hydrosilylation of aromatic alkenes. J. Am. Chem. Soc. 124, 4558-4559 (2002).

23. Jia, X. \& Huang, Z. Conversion of alkanes to linear alkylsilanes using an iridium-iron-catalysed tandem dehydrogenation-isomerization-hydrosilylation. Nat. Chem. 8, 157-161 (2016).

24. Atienza, C. C. H. et al. Bis(imino)pyridine cobalt-catalyzed dehydrogenative silylation of alkenes: scope, mechanism, and origins of selective allylsilane formation. J. Am. Chem. Soc. 136, 12108-12118 (2014).
25. Noda, D., Tahara, A., Sunada, Y. \& Nagashima, H. Non-precious-metal catalytic systems involving iron or cobalt carboxylates and alkyl isocyanides for hydrosilylation of alkenes with hydrosiloxanes. J. Am. Chem. Soc. 138, 2480-2483 (2016).

26. Buslov, I., Becouse, J., Mazza, S., Montandon-Clerc, M. \& Hu, X. Chemoselective alkene hydrosilylation catalyzed by nickel pincer complexes. Angew. Chem. Int. Ed. 54, 14523-14526 (2015).

27. Docherty, J. H., Peng, J., Dominey, A. P. \& Thomas, S. P. Activation and discovery of earth-abundant metal catalysts using sodium tert-butoxide. Nat. Chem. 9, 595-600 (2017)

28. Fürstner, A. et al. Preparation, structure, and reactivity of nonstabilized organoiron compounds. Implications for iron-catalyzed cross coupling reactions. J. Am. Chem. Soc. 130, 8773-8787 (2008).

29. Bedford, R. B. How low does iron go? Chasing the active species in Fe-catalyzed cross-coupling reactions. Acc. Chem. Res. 48, 1485-1493 (2015).

30. Daifuku, S. L., Kneebone, J. L., Snyder, B. E. R. \& Neidig, M. L. Iron(II) active species in iron-bisphosphine catalyzed Kumada and Suzuki-Miyaura crosscouplings of phenyl nucleophiles and secondary alkyl halides. J. Am. Chem. Soc. 137, 11432-11444 (2015).

31. Collins, K. D. \& Glorius, F. A robustness screen for the rapid assessment of chemical reactions. Nat. Chem. 5, 597-601 (2013).

32. Wang, C., Teo, W. J. \& Ge, S. Cobalt-catalyzed regiodivergent hydrosilylation of vinylarenes and aliphatic alkenes: ligand- and silane-dependent regioselectivities. ACS Catal. 7, 855-863 (2017).

33. Cheng, B., Lu, P., Zhang, H.-Y., Cheng, X.-P. \& Lu, Z. Highly enantioselective cobalt-catalyzed hydrosilylation of alkenes. J. Am. Chem. Soc. 139, 9439-9442 (2017).

34. Fontaine, F.-G. \& Zargarian, D. $\mathrm{Me}_{2} \mathrm{AlCH}_{2} \mathrm{PMe}_{2}$ : a new, bifunctional cocatalyst for the $\mathrm{Ni}(\mathrm{II})$-catalyzed oligomerization of $\mathrm{PhSiH}_{3}$. J. Am. Chem. Soc. 126, 8786-8794 (2004).

35. Chen, Y., Sui-Seng, C., Boucher, S. \& Zargarian, D. Influence of $\mathrm{SiMe}_{3}$ substituents on structures and hydrosilylation activities of $\left(\left(\mathrm{SiMe}_{3}\right)_{1 \text { or2 }}\right.$-indenyl) $\mathrm{Ni}\left(\mathrm{PPh}_{3}\right) \mathrm{Cl}$. Organometallics 24, 149-155 (2005).

36. Chen, Y. \& Zargarian, D. Phenylsilane dehydrocoupling and addition to styrene catalyzed by (R-indenyl)Ni(phosphine)(methyl) complexes. Can. J. Chem. 87, 280-287 (2009)

37. Junquera, L. B., Puerta, M. C. \& Valerga, P. R-allyl nickel(II) complexes with chelating $\mathrm{N}$-heterocyclic carbenes: synthesis, structural characterization, and catalytic cctivity. Organometallics 31, 2175-2183 (2012).

38. Espinal-Viguri, M., Woof, C. R. \& Webster, R. L. Iron-catalyzed hydroboration: unlocking reactivity through ligand modulation. Chem. Eur. J. 22, 11605-11608 (2016).

39. MacNair, A. J., Millet, C. R. P., Nichol, G. S., Ironmonger, A. \& Thomas, S. P. Markovnikov-selective, activator-free iron-catalyzed vinylarene hydroboration. ACS Catal. 6, 7217-7221 (2016).

40. Chen, X., Cheng, Z. \& Lu, Z. Iron-catalyzed, Markovnikov-selective hydroboration of styrenes. Org. Lett. 19, 969-971 (2017).

41. Palmer, W. N., Diao, T., Pappas, I. \& Chirik, P. J. High-activity cobalt catalysts for alkene hydroboration with electronically responsive terpyridine and $\alpha$-diimine ligands. ACS Catal. 5, 622-626 (2015).

42. Scheuermann, M. L., Johnson, E. J. \& Chirik, P. J. Alkene isomerizationhydroboration promoted by phosphine-ligated cobalt catalysts. Org. Lett. 17, 2716-2719 (2015).

43. Guo, J. \& Lu, Z. Highly chemo-, regio-, and stereoselective cobalt-catalyzed Markovnikov hydrosilylation of alkynes. Angew. Chem. Int. Ed. $\mathbf{5 5}$ 10835-10838 (2016).

44. Peng, J., Docherty, J. H., Dominey, A. P. \& Thomas, S. P. Cobalt-catalysed Markovnikov selective hydroboration of vinylarenes. Chem. Commun. 53, 4726-4729 (2017). 
45. Greenhalgh, M. D., Frank, D. J. \& Thomas, S. P. Iron-catalysed chemo-, regio-, and stereoselective hydrosilylation of alkenes and alkynes using a bench-stable iron(II) pre-catalyst. Adv. Synth. Catal. 356, 584-590 (2014).

46. Lee, H. et al. Mechanistic insight into high-spin iron(I)-catalyzed butadiene dimerization. Organometallics 35, 2923-2929 (2016).

\section{Acknowledgements}

We thank the National Natural Science Foundation of China (21625204, 21421062, 21290182, and 21702109), the National Basic Research Program of China

(2012CB821600), the "111" project (B06005) of the Ministry of Education of China, the National Program for Support of Top-notch Young Professionals, "1000-Talent Youth Plan", and the European Community (FP7-PEOPLE-2012-IIF under grant agreement 912364 to Q.P.) for financial support. We thank Prof. Wei Shi for his generous help on analysis of magnetic moments and his discussion on EPR data.

\section{Author contributions}

S.-F.Z. and M.-Y.H. conceived the research program and designed and directed the investigations. M.-Y.H. carried out the hydrosilylation reactions. M.-Y.H., Q.H., Z.-C.W., L.-Y.L. and Y.-J.M. prepared the ligands and substrates. Q.P. and S.-J.F. proposed and modeled the reaction mechanism through the computational study. S.-F.Z., Q.P. and M.-Y.H. wrote the manuscript.

\section{Additional information}

Supplementary Information accompanies this paper at https://doi.org/10.1038/s41467017-02472-6.
Competing interests: The authors declare no competing financial interests.

Reprints and permission information is available online at http://npg.nature.com/ reprintsandpermissions/

Publisher's note: Springer Nature remains neutral with regard to jurisdictional claims in published maps and institutional affiliations.

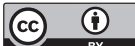

Open Access This article is licensed under a Creative Commons Attribution 4.0 International License, which permits use, sharing, adaptation, distribution and reproduction in any medium or format, as long as you give appropriate credit to the original author(s) and the source, provide a link to the Creative Commons license, and indicate if changes were made. The images or other third party material in this article are included in the article's Creative Commons license, unless indicated otherwise in a credit line to the material. If material is not included in the article's Creative Commons license and your intended use is not permitted by statutory regulation or exceeds the permitted use, you will need to obtain permission directly from the copyright holder. To view a copy of this license, visit http://creativecommons.org/ licenses/by/4.0/

(C) The Author(s) 2018 